\title{
A SEMIANALYTICAL METHOD TO SOLVE ALTARELLI-PARISI EVOLUTION EQUATIONS
}

\author{
P. SANTORELLI* E. SCRIMIERI \\ ${ }^{a}$ Dipartimento di Scienze Fisiche, Università "Federico II" di Napoli \\ Mostra d'Oltremare, Pad. 20, I-80125 Napoli, and INFN, Sezione di Napoli, Italy \\ ${ }^{b}$ Dipartimento di Fisica, Università di Bari, \\ Via G. Amendola, 173, I-70126 Bari, and INFN, Sezione di Bari, Italy.
}

Abstract: We discuss a new method to solve in a semianalytical way the Dokshitzer-Gribov-Lipatov-Altarelli-Parisi evolution equations at NLO order in the $x$-space. The method allows to construct an evolution operator expressed in form of a rapidly convergent series of matrices, depending only on the splitting functions. This operator, acting on a generic initial distribution, provides a very accurate solution in a short computer time (only a few hundredth of second). As an example, we apply the method, useful to solve a wide class of systems of integrodifferential equations, to the polarized parton distributions.

\section{Introduction}

The scaling violation of nucleon structure functions is described in terms of Dokshitzer-GribovLipatov-Altarelli-Parisi (DGLAP) evolution equations $[\overline{1} 1$. . The DGLAP integrodifferential equations describe the $Q^{2}$ dependence of the structure functions, which are related, via the operator product expansion, to the parton distributions for which the DGLAP equations are usually written down. In this framework, the analysis of the experimental data, is performed fixing at some $Q_{0}^{2}$ the structure functions by assuming the parton distributions and computing the convolution with the coefficient functions, which can be evaluated in perturbation theory. The comparison with experimental data, which are distributed at different values of $Q^{2}$, goes through the solution of DGLAP equations for the parton distributions; thus a reliable and fast algorithm to solve these equations is welcome.

In literature there are essentially three different approaches to solve the DGLAP equations. The first one is based on the Laguerre polynomials expansion [2]. This technique is quite accu-

${ }^{*}$ Conference speaker rate up to $x$-values not smaller than $\bar{x} \approx 10^{-3}$; on the contrary, below $\bar{x}$ the convergence of the expansion slows down $[\overline{3}, \overline{4}, \overline{4}|\overline{1}|$. Given that experimental data are already available down to about $\bar{x}$, for the polarized case, and down to $10^{-5}$, for the unpolarized case, this method results no longer practical.

An alternative approach takes advantage of the fact that the moments of the convolutions appearing in the equations factorize in such a way that the analytical solution, in the momentum space, can be written down [i5]. However, also in the most favorable case in which the analytical expressions of the moments of the initial conditions are known, the numerical Mellin inversion is relatively CPU time consuming (see $\overline{6}|\overline{1}|$ ). Moreover, as discussed in $[\overline{7} \mid$, since $x$ variable is related to the invariant energy $W^{2}$ of the virtual photonhadron scattering process by $W^{2}=(1-x) / x$, $x \rightarrow 0$ is the infinite energy limit and thus can never experimentally be reached. As a consequence of this all moments are plagued by an a priori infinite uncertainty, which can be reduced by means of assumptions implying that any use of the evolution equations for moments is model dependent. The more simple solution to 
this problem is to solve DGLAP equations in the $x$-space. In this framework, besides the Laguerre method, another strategy, the so called "brute force" method [i], represents a good candidate. It is fundamentally a finite-differences method of solution which reaches a good precision in the

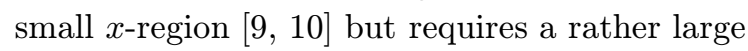
amount of computer running time.

Here we discuss a semianalytical method, in the $x$-space, to solve DGLAP equations [11 1, It consists in constructing an evolution operator which, depending only on the splitting functions, can be worked out once for all. In this respect our strategy is similar to the one in $\left[\begin{array}{cc}\overline{2} & \overline{2} \\ 2 & \overline{1} \\ 1\end{array}\right]$. Our method to perform the convolutions instead, takes advantage of an $x$ discretization (comparable to the one in $\left[\overline{9}_{1}^{\prime}, 10_{1}^{n}\right)$ which allows us to represent the evolution operator as a matrix. Thus the procedure to construct the solution reduces merely to a multiplication between the evolution matrix and an initial vector, and can be done in an extremely short computer time with the required accuracy. This is particularly appealing in the analysis of the experimental data on nucleon structure functions which requires a large number of parton evolutions.

In the next section we discuss the (formal) analytical solution of the DGLAP equations; in the third one the algorithm to perform the $x$-integration is presented. The last two sections are devoted to analyze the numerical results relative to the evolution of polarized parton distributions, to study the yield of our method in comparison with others and to conclude.

\section{The Evolution Operator}

The DGLAP equation, up to Next-to-LeadingOrder (NLO) corrections, for the Non-Singlet distribution is*

$$
\begin{aligned}
& \frac{\partial}{\partial t} \quad \Delta \tilde{q}_{N S}(x, t)= \\
& \left(\Delta \tilde{P}_{N S}^{(0)}(x)+\alpha(t) \Delta \tilde{R}_{N S}(x)\right) \otimes \Delta \tilde{q}_{N S}\left(x, t_{\chi}(2.1)\right.
\end{aligned}
$$

\footnotetext{
*In the following we limit ourselves to discuss the polarized parton distributions. The application of our method to the unpolarized case is straightforward.
}

while for the Singlet and Gluon distributions we have:

$$
\begin{aligned}
& \frac{\partial}{\partial t}\left(\begin{array}{c}
\Delta \tilde{q}_{S}(x, t) \\
\Delta \tilde{g}(x, t)
\end{array}\right)= \\
& \left(\begin{array}{cc}
\Delta \tilde{P}_{q q}^{(0)}(x) & \Delta \tilde{P}_{q g}^{(0)}(x) \\
\Delta \tilde{P}_{g q}^{(0)}(x) & \Delta \tilde{P}_{g g}^{(0)}(x)
\end{array}\right) \otimes\left(\begin{array}{c}
\Delta \tilde{q}_{S}(x, t) \\
\Delta \tilde{g}(x, t)
\end{array}\right)+ \\
& \alpha(t)\left(\begin{array}{cc}
\Delta \tilde{R}_{q q}(x) & \Delta \tilde{R}_{q g}(x) \\
\Delta \tilde{R}_{g q}(x) & \Delta \tilde{R}_{g g}(x)
\end{array}\right) \otimes\left(\begin{array}{c}
\Delta \tilde{q}_{S}(x, t) \\
\Delta \tilde{g}(x, t)
\end{array}\right)^{2, .2}
\end{aligned}
$$

where

$$
\Delta \tilde{R}_{i j}(x) \equiv \Delta \tilde{P}_{i j}^{(1)}(x)-\frac{\beta_{1}}{2 \beta_{0}} \Delta \tilde{P}_{i j}^{(0)}(x) .
$$

In these equations the symbol $\otimes$ stands for

$$
f(x) \otimes g(x) \equiv \int_{x}^{1} \frac{d y}{y} f\left(\frac{x}{y}\right) g(y)
$$

and

$$
\tilde{f}(x) \equiv x f(x) .
$$

Instead of $Q^{2}$, we have used the variable $t$ defined by

$$
t=-\frac{2}{\beta_{0}} \ln \left[\frac{\alpha_{s}\left(Q^{2}\right)}{\alpha_{s}\left(Q_{0}^{2}\right)}\right],
$$

where $\alpha_{s}$ is strong running coupling constant corrected at $\mathrm{NLO}$ :

$$
\alpha_{s}\left(Q^{2}\right)=\frac{4 \pi}{\beta_{0} \ln \left(Q^{2} / \Lambda^{2}\right)}\left[1-\frac{\beta_{1} \ln \left(\ln \left(Q^{2} / \Lambda^{2}\right)\right)}{\beta_{0}^{2} \ln \left(Q^{2} / \Lambda^{2}\right)}\right]
$$

and so in Eqs. $(\overline{2} . \overline{1} \cdot \overline{1})-(\underline{2} \cdot \overline{2})$

$$
\alpha(t) \equiv \frac{\alpha_{s}\left(Q_{0}^{2}\right)}{2 \pi} \operatorname{Exp}\left\{-\frac{\beta_{0}}{2} t\right\} .
$$

The explicit expressions for $\beta_{0}, \beta_{1}$ as well as for the Splitting Functions $\Delta P_{i j}(x)$ can be found in [12in].

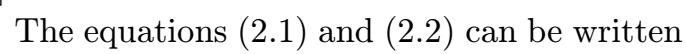
in the following general form:

$$
\frac{\partial}{\partial t} \mathbf{f}(t)=\boldsymbol{\Omega}(t) \odot \mathbf{f}(t)
$$

where $\mathbf{f}(t)$ indicates the "vector of components $f(x, t)$ " and $\boldsymbol{\Omega}(t)$ a linear operator acting as:

$$
[\boldsymbol{\Omega}(t) \odot \mathbf{f}(t)]_{x} \equiv \int_{x}^{1} d y \omega(x, y, t) f(y, t) .
$$

Note that, in the Singlet-Gluon case, $\mathbf{f}(t)$ becomes a doublet of vectors and $\boldsymbol{\Omega}(t)$ a 2 × 2 matrix of operators. 
Due to the logarithmic dependence of $t$ on $Q^{2}$, the range of values of physical interest for $t-t_{0}\left(t_{0}\right.$ is the starting values of $t$, where the parton distributions are assumed known) is small enough to expect that the Taylor's series of the solution $\mathbf{f}(t)$ converges rapidly. On the other hand, by deriving repeatedly the Eq. (2.9i) we can write:

$$
\left.\frac{\partial^{k}}{\partial t^{k}} \mathbf{f}(t)\right|_{t=t_{0}}=\mathbf{M}^{(k)} \odot \mathbf{f}\left(t_{0}\right)
$$

where the operators $\mathbf{M}^{(k)}$ can be obtained recursively:

$$
\begin{aligned}
\mathbf{M}^{(0)} & =\mathbf{I} \\
\mathbf{M}^{(1)} & =\boldsymbol{\Omega}_{0} \\
\mathbf{M}^{(2)} & =\boldsymbol{\Omega}_{0}^{(1)}+\boldsymbol{\Omega}_{0} \odot \mathbf{M}^{(1)} \\
\mathbf{M}^{(3)}= & \boldsymbol{\Omega}_{0}^{(2)}+2 \boldsymbol{\Omega}_{0}^{(1)} \odot \mathbf{M}^{(1)}+\boldsymbol{\Omega}_{0} \odot \mathbf{M}^{(2)} \\
\ldots & \ldots \ldots . \\
& \quad \ldots \ldots \\
\mathbf{M}^{(k)}= & \sum_{i=0}^{k-1} c_{i}^{(k)} \boldsymbol{\Omega}_{0}^{(k-1-i)} \odot \mathbf{M}^{(i)} .
\end{aligned}
$$

The $c_{i}^{(k)}$ indicates the $i$-th term of the $k$-th row of Tartaglia triangle and

$$
\boldsymbol{\Omega}_{0} \equiv \boldsymbol{\Omega}\left(t_{0}\right),\left.\quad \boldsymbol{\Omega}_{0}^{(k)} \equiv \frac{\partial^{k}}{\partial t^{k}} \boldsymbol{\Omega}(t)\right|_{t=t_{0}}
$$

Then the solution can be written as:

$$
\begin{aligned}
\mathbf{f}(t) & =\left(\sum_{k=0}^{\infty} \frac{\left(t-t_{0}\right)^{k}}{k !} \mathbf{M}^{(k)}\right) \odot \mathbf{f}\left(t_{0}\right) \\
& \equiv \mathbf{T}\left(t-t_{0}\right) \odot \mathbf{f}\left(t_{0}\right)
\end{aligned}
$$

with $\mathbf{T}\left(t-t_{0}\right)$ the Evolution Operator. As we

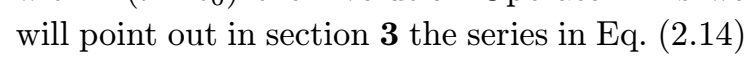
converge quickly enough to obtain a very good approximation retaining only a first few terms. It is worth to note that if the operator $\boldsymbol{\Omega}(t)$ can be written as $h(t) \Omega^{\prime}$ (with $h(t)$ a numerical function) it is easy to show that the series in Eq. (12.14) reduces to:

$$
\mathbf{f}(t)=\operatorname{Exp}\left\{\left[\int_{t_{0}}^{t} h(\tau) d \tau\right] \boldsymbol{\Omega}^{\prime}\right\} \odot \mathbf{f}\left(t_{0}\right) .
$$

This is the case of DGLAP equation at Leading Order (LO) approximation. Nevertheless, in Eqs. (2.1)-(2.2i), where NLO corrections are included, we have $\boldsymbol{\Omega}(t)=\boldsymbol{\Omega}_{1}+\alpha(t) \boldsymbol{\Omega}_{2}$, with $\boldsymbol{\Omega}_{1}$ and $\boldsymbol{\Omega}_{2}$ non-commuting operators. As a consequence the series in Eq. (12.1- $\overline{4}$ ) cannot be summed and it is not possible write the solution in a closed form.

\section{The $x$-Integration}

The integrals in Eq. (2.4) are evaluated with a method that generalizes the one proposed in Ref. [101]. The method consists to treat exactly the "bad" behaviour of the kernel $\omega(x, y, t)$ in Eq. (2.10") and approximate the "smooth" function $f(y, \bar{t})$. In particular, we construct a $M+1$ points grid $\left(x_{0}>0, x_{1}, \ldots, x_{M-1}, x_{M}=1\right)$ in the interval ] $0,1]$ and approximate $f(x)$ in each interval $\left[x_{k}, x_{k+1}\right]$ by the cubic which fits the four point $f\left(x_{i}\right)$, with $i=k-1, k, k+1, k+2$ :

$$
f(x) \approx \sum_{l=1}^{4} a_{l}^{(k)} x^{l-1}(x) \quad \forall x \in\left[x_{k}, x_{k+1}\right] .
$$

The general structure of the Polarized Splitting Functions which appear in Eqs. (2.1)

$$
\Delta \tilde{P}(x)=\frac{\mathcal{A}(x)}{(1-x)_{+}}+\mathcal{B}(x)+\delta(1-x) \mathcal{C},
$$

and therefore the " $i$ component" of the convolution is:

$$
\begin{aligned}
& \Delta \tilde{P}\left(x_{i}\right) \otimes f\left(x_{i}\right)= \\
& x_{i}\left(\int_{x_{i}}^{1} \frac{d y}{y} \frac{\mathcal{A}\left(x_{i} / y\right) f(y)-\mathcal{A}(1) f\left(x_{i}\right)}{y-x_{i}}+\right. \\
& \left.\quad \int_{x_{i}}^{1} \frac{d y}{y^{2}} \mathcal{B}\left(\frac{x_{i}}{y}\right) f(y)\right)+ \\
& \left(\mathcal{C}+\mathcal{A}(1) \ln \left(1-x_{i}\right)\right) f\left(x_{i}\right) .
\end{aligned}
$$

Substituting Eq. (30.1) in Eq. $(\overrightarrow{3} .3)$ we obtain $\forall i \in$ $\{0, \ldots, M-1\}\left(\sum_{k=M}^{M-1} \equiv 0\right.$ is understood $)$

$$
\begin{aligned}
& \Delta \tilde{P}\left(x_{i}\right) \otimes f\left(x_{i}\right)= \sum_{l=1}^{m} a_{l}^{(i)}\left(\beta_{l}^{i}+\rho_{i l}^{i}\right)+ \\
& \sum_{k=i+1}^{M-1} \sum_{l=1}^{m} a_{l}^{(k)}\left(\gamma_{k l}^{i}+\rho_{k l}^{i}\right)+ \\
&\left(\mathcal{C}+\mathcal{A}(1) \ln \left(1-x_{i}\right)-\mathcal{A}(1) \sigma^{i}\right) f\left(x_{i}\right) ;
\end{aligned}
$$

†The same structure, however, holds for unpolarized and transversely polarized splitting functions. 
then we have:

$$
\Delta \tilde{P}\left(x_{i}\right) \otimes f\left(x_{i}\right)=\sum_{k=0}^{M} \omega_{i k} f\left(x_{k}\right),
$$

where $\omega$ is the matrix of the coefficients of $f\left(x_{k}\right)$. The analytical expressions for the matrices $\beta, \gamma$, $\rho$ and $\sigma$ can be found in [i] 1 in.

Therefore the Eqs. $(2,1,1)-(22.2)$ became ${ }^{\ddagger}$ :

$$
\begin{aligned}
& \frac{\partial}{\partial t} \Delta \tilde{q}_{N S}\left(x_{i}, t\right)= \\
& \sum_{k=0}^{M}\left(\omega_{i k}^{(0) N S}+\alpha(t) \omega_{i k}^{(1) N S}\right) \Delta \tilde{q}_{N S}\left(x_{k}, t\right)(3.6)
\end{aligned}
$$

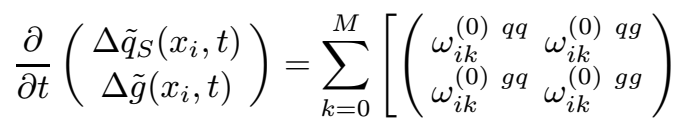

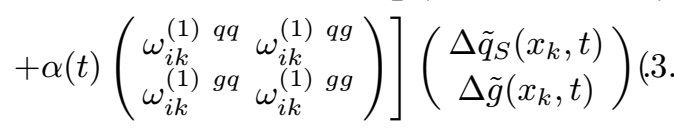

We solve these equations by means of the method shown in section $2_{i}^{-i}$ the operator $\Omega(t)$ and then the $\mathbf{M}^{(k)}$ became now numerical matrices, and the symbol $\odot$ stands for the usual rows by columns product. We would stress the fact that the matrices $\mathbf{M}^{(k)}$ depend only on the points $x_{i}$ and so they can be numerically evaluated once for all.

\section{Numerical Analysis}

The convergence of our algorithm is controlled by two parameters: the order $n$ of the truncated series

$$
\mathbf{T}^{(n)}\left(t-t_{0}\right)=\sum_{k=0}^{n} \frac{\left(t-t_{0}\right)^{k}}{k !} \mathbf{M}^{(k)},
$$

which define the evolution operator, and the number $M$ of the points of $x$-integration.

To test the accuracy of our method we evolve the Gehrmann and Stirling polarized singlet-gluon initial distributions (cf [1] $\left(t_{0}=0\right)$ to $Q^{2}=200 \mathrm{GeV}^{2}(t=0.136)$ and $Q^{2}=50000 \mathrm{GeV}^{2}(t=0.245)$. We choose to work, as in the paper $[\overline{9}]$, in the fixed flavour scheme, $n_{f}=3$, with $\Lambda_{Q C D}^{(4)}=231 \mathrm{MeV}$, and

\footnotetext{
$\ddagger$ Note that $\omega^{(1)}$ matrices correspond to the convolutions of the parton distributions with $\Delta \tilde{R}$ (cf Eq. $(2.3)$ ).
}

without taking into account, in the $Q^{2}$ evolution of $\alpha_{s}$, quark thresholds. The range ]0,1] has been divided in $M$ steps by $M+1$ points: $x_{0}, x_{1}, \ldots, x_{M}$ distributed in such a way that the function $\ln (x)+2 x$ varies by the same amount at any step; this function is slightly different from the pure logarithmic distribution commonly used in literature $\left.{ }^{[9,}, 10_{1}^{\prime}\right]$, but allow, in our case, a more uniform distribution of the numerical errors. The end points are fixed to be $x_{0}=1 \times 10^{-8}$ and $x_{M}=1$; however, for a better reading, in the Figures '1-i-is the $x$-axis ranges from $10^{-4}$ to 1 .

First, we fix $M=100$. In Figs. '1i-i reported the evolved singlet and gluon distributions, respectively, obtained with $n=3,6$ and 12 for $Q^{2}=200 \mathrm{GeV}^{2}$ and $Q^{2}=50000 \mathrm{GeV}^{2}$. It is worth to note the very fast convergence of the series to the solution, as already observed above. As a matter of fact, the maximum difference between the solutions relative to $n=6$ and $n=12$ is $1.4 \times 10^{-5}\left(7.6 \times 10^{-4}\right)$ for the singlet, and $9.3 \times 10^{-5}\left(5.3 \times 10^{-3}\right)$ for the gluon distribution, in correspondence of $Q^{2}=200 \mathrm{GeV}^{2}\left(Q^{2}=\right.$ $50000 \mathrm{GeV}^{2}$ ).

Next we fix $n=12$ and $Q^{2}=200 \mathrm{GeV}^{2}$. In Figs. 's-i are plotted the approximated evolved distributions with $M=25,50,100$ : the maximum difference on the common points between $M=50$ and $M=100$ is $4.6 \times 10^{-4}$ for the singlet and $7.9 \times 10^{-4}$ for the gluons. By comparing the results in Fig. '3- $\overline{3}$ ' with the corresponding Figs. 1-4 in Ref. [9], we observe, besides a good numerical agreement of the results, a faster convergence as the number $\mathrm{M}$ of integration points increases, as a consequence of our more accurate $x$-integration procedure with respect to the so called "brute force" methods. In fact it should be observed that reducing from the cubic to the linear approximation of $f(x)$ in Eq. (3. $\overline{1}$. accuracy $\mathcal{E}(x, t)$ (defined in the sequel) becomes about 1 and 3 order of magnitude bigger, respectively for singlet and gluon.

To discuss the degree of accuracy of the method in [11 1, were introduced a global accuracy $\mathcal{E}(x, t)$ defined as the difference between left and righthand side of the Eq. (2.2i). The comparison between the range of values of $\mathcal{E}$ with the one of both sides of Eq. (2.2i) represents a very good estimate of the degree of accuracy of the solution. 


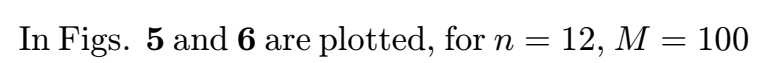
and $Q^{2} \stackrel{-1}{=} 200 \mathrm{GeV}^{2}$ both sides of the Eq. (2.2.2) and the corresponding (rescaled) accuracy $\mathcal{E}(x, t)$ $\S$. It appears evident that an excellent approximation of the solution is obtained.

Another advantage of our method, once fixed the accuracy of the solution, appears to be the running time to get each evolution. In fact, the simple analytical structure of the evolution matrix $\mathbf{T}$ makes the solution procedure considerably fast. As a matter of fact, once given the Splitting Functions and constructed the corresponding matrices $\mathbf{M}^{(k)}$ (we have used Mathematica [1] 14 in to this), a single evolution, i.e. the multiplication of the $\mathbf{T}$ evolution matrix by the initial vector, require, for $n=12$ and $M=100$, about $6 \times 10^{-2}$ sec on an AlphaServer 1000 using a Fortran Code.

Particularly interesting is the comparison between our method and the one presented in [2, ind , where an evolution operator is also introduced. Firstly we observe that the latter method is based on a polynomial expansion of the splitting and distribution functions. The expansion is equivalent to an expansion in power of $x$. As a consequence it is affected by problems of convergence for $x \rightarrow 0$, due to the branch point in zero of the involved functions. This is the source of the difficult encountered in the small- $x$ region, which are not present in our approach in which an optimized Newton-Cotes-like quadrature formula is employed.

Second, also in the $x$-region of convergence, the Laguerre polynomial expansion need, for each evolution process, the computation of the moments of the initial parton distributions with respect to the polynomials: this procedure requires a remarkable amount of CPU-time with respect to our approach in which only the evaluation of the initial parton distribution in the $M$ grid points is needed.

\footnotetext{
$\S$ The integration in the right-hand side has been performed numerically after an $x$-interpolation of the discrete values obtained with the evolution operator, while the left-hand side is worked out by direct derivation of Eq. (12.14).
}

\section{Conclusions}

We have discussed a new algorithm to solve the DGLAP evolution equations, in the $x$-space, which appears suitable for a rather large class of coupled integrodifferential equations.

The method produces a solution which is analytical in the $Q^{2}$-evolution parameter and approximate, but rapidly convergent, in the $x$-space. It allows to construct, once for all, an evolution operator in matrix form. It depends only on the splitting functions appearing in the equations and can be rapidly applied to whatever initial distribution to furnish the evolved one, requiring for each evolution only a few hundredth of second.

It is worth to note the reliability of our $x^{-}$ integration algorithm, which gets excellent approximations on the whole $x$-range (we use, for all the calculations, $10^{-8} \leq x \leq 1$ ), also with few integration points, resulting in an evolution matrix of particularly small dimensions.

In conclusion, our method, whose numerical implementation is straightforward, appears to be very fast, very accurate and extremely stable with respect to the increasing of convergence parameters (i.e. $n$, the order of the truncated series which gives the Evolution Operator, and $M$, the number of integration points). For these reasons it represents a powerful tool to analyze the experimental data on nucleon structure functions.

\section{Acknowledgments}

One of us (P.S.) thanks G. Altarelli for useful discussions during the Corfú Conference.

\section{References}

[1] V.N. Gribov and L.N. Lipatov, Sov. J. Nucl. Phys. 15 (1972) 428; G. Altarelli and G. Parisi, Nucl. Phys. B126 (1977) 298; Yu.L. Dokshitzer, Sov. Phys. JETP 46 (1977) 641.

[2] W. Furmanski and R. Petronzio, Nucl. Phys. B195 (1982) 237; W. Furmanski and R. Petronzio, Z. Phys. C11 (1982) 293; G.P. Ramsey, J. Comp. Phys. 60 (1992) . 
[3] S. Kumano and J.T. Londergan, Comput. Phys. Commun. 69 (1992) 373.

[4] C. Corianò and C. Savkli, ihep-ph/9803336!

[5] M. Glück, E. Reya, and A. Vogt, Z. Phys. C48 (1990) 471; Phys. Rev. D45 (1992) 3986.

[6] B. Adeva et al. (SMC Collaboration), CERNEP/98-86.

[7] S. Forte, and L. Magnea, Phys. Lett. B448 (1999) 295

[8] G.P. Ramsey, PhD Thesis, Illinois Institute of Technology, 1982; M. Miyama, and S. Kumano, Comput. Phys. Commun. 94 (1996) 185.

[9] M. Hirai, S. Kumano, and M. Miyama, Comput. Phys. Commun. 108 (1998) 38.

[10] D. Fasching, hep-ph/9610261'.

[11] P. Santorelli, and E. Scrimieri, 'hep-ph/9807572i, will appear in Phys. Lett. B.

[12] R. Mertig and W.L. van Neerven, Z. Phys. C70 (1996) 637; W. Vogelsang, Phys. Rev. D54 (1996) 2023; cf also Ref. [i9i]

[13] T. Gehrmann and V.J. Stirling, Phys. Rev. D53 (1996) 6100.

[14] S. Wolfram, The Mathematica Book, 3rd ed. (Wolfram Media/Cambridge University Press, 1996).

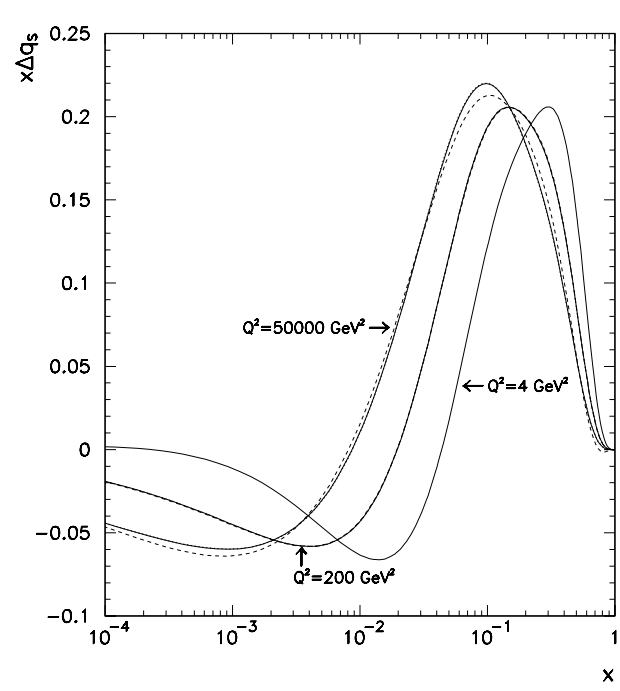

Figure 1: The initial Singlet distribution $\left(Q^{2}=\right.$ $4 \mathrm{GeV}^{2}$, solid line) and the evolved ones for $n=$ 3 (dashed lines), $n=6$ (dotted lines) and $n=12$ (solid lines) corresponding at $Q^{2}=200 \mathrm{GeV}^{2}$ and $Q^{2}=50000 \mathrm{GeV}^{2}$. We use $M=100$.

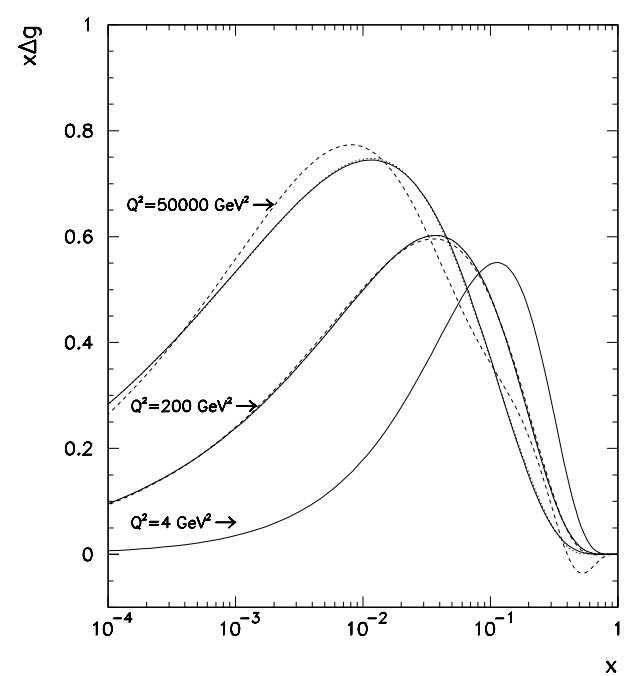

Figure 2: The same in Fig. 긴 for Gluons. 


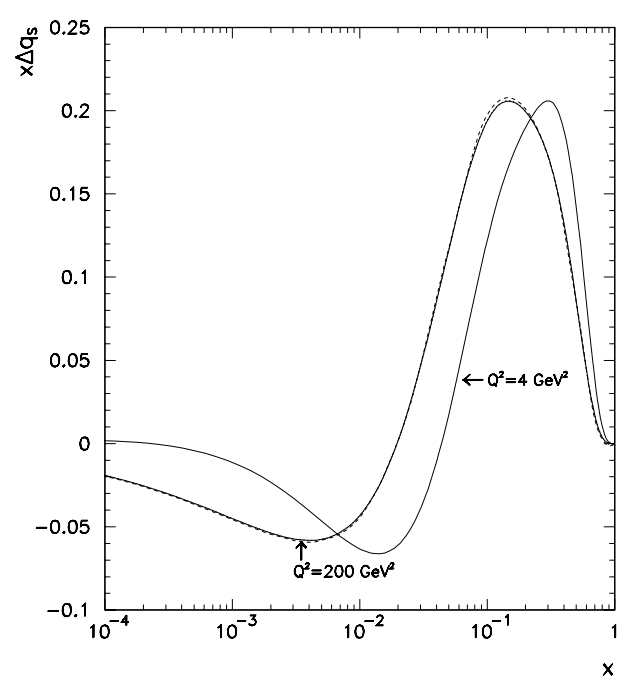

Figure 3: The initial Singlet distribution $\left(Q^{2}=\right.$ $4 \mathrm{GeV}^{2}$, solid line) and the evolved one at $Q^{2}=$ $200 \mathrm{GeV}^{2}$ with $M=100$ (solid line), $M=50$ (dotted line) and $M=25$ (dashed line) with $n=12$.

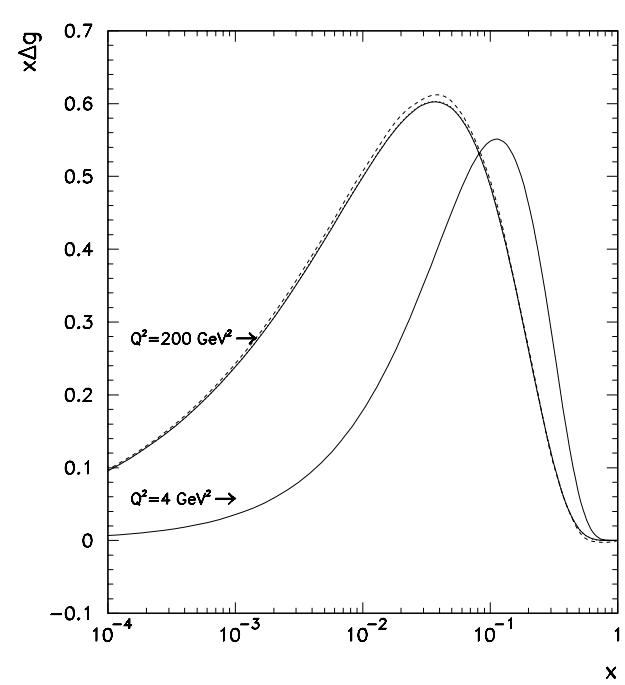

Figure 4: The same in Fig. $\overline{3}, 1$ for Gluons.

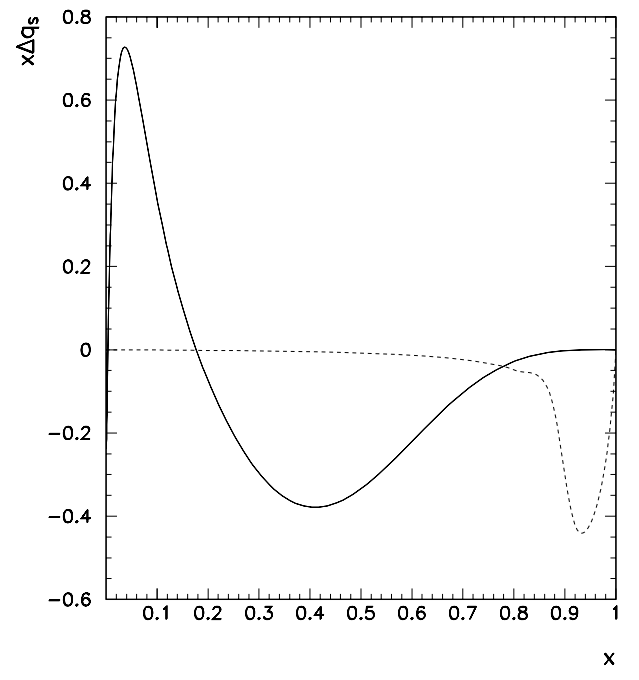

Figure 5: For $n=12, M=100$ and $Q^{2}=200 \mathrm{GeV}^{2}$ both sides of the Eq. (2.2.7) are plotted (solid line and dotted line), in correspondence of the Singlet distribution. Dashed line represents $\mathcal{E}(x) \times 10^{3}$ (see text).

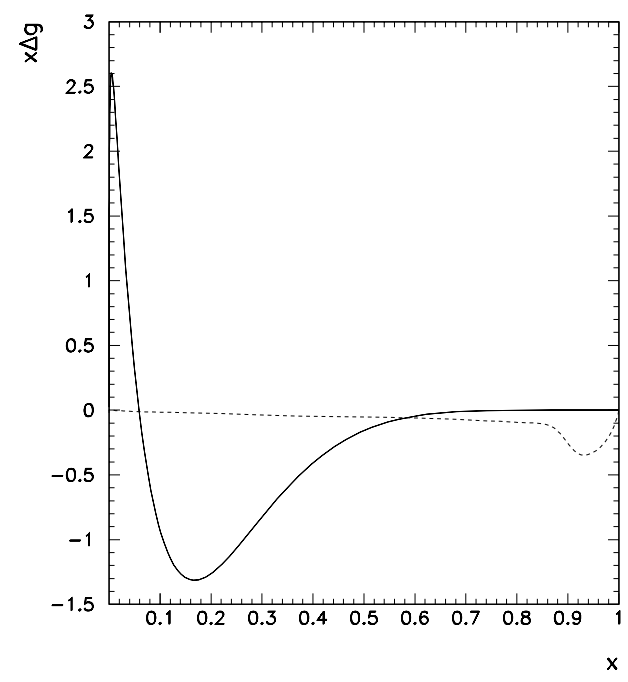

Figure 6: The same in Fig. 5 for Gluons. Dashed line represents $\mathcal{E}(x) \times 10^{4}$ (see text). 